\title{
Tire-wear control in aircraft via active braking
}

\author{
L. D’Avico ${ }^{1}$, M. Tanelli ${ }^{1,2}$, S.M. Savaresi ${ }^{1}$
}

\begin{abstract}
In ground vehicles, tire consumption is in general mainly due to the mileage covered, and in fact the life span of tires, at least in common situations, is rather long. In the aeronautical context, and for aircraft in particular, instead, tire consumption plays a crucial role in determining the maintenance costs. This is due to the fact that, in aircraft braking, nearly all maneuvers activate the anti-skid controller, which remains in use for long time intervals. In ground vehicles, instead ABS systems are usually active for short time intervals which cover a part of the braking maneuvers only. Thus, tire consumption in the automotive context is usually studied under constant speed assumptions. In this work, we formulate a tire consumption model that encompasses explicitly the wheel acceleration/deceleration dynamics, and we show that tire wear can be directly related to the anti-skid controller parameters. Based on this, a sensitivity analysis of tire-consumption versus braking performance is carried out, showing that, using an appropriate anti-skid control approach, one may directly formulate the braking problem as a tire consumption regulation one, being sure that the resulting braking performance will have an a priori guaranteed outcome. The tire wear model is also validated in an experimental setting.
\end{abstract}

Index Terms-Tire-wear; aircraft; anti-skid; braking control; landing gear.

\section{INTRODUCTION}

Historically, anti-skid controllers have been first developed for aircraft braking systems. However, to the best of the authors' knowledge, a very limited scientific literature is available on this topic. In the current industrial practice, antiskid systems are mainly provided by braking systems suppliers as black-boxes that come with the actuation system, and which only need connection with the wheel speed sensor. They are installed in the landing gear, and each landing gear has a dedicated anti-skid system which might in some cases share limited information with the other local anti-skids (for example to gather the values of all the braked-wheel speeds), but it does not in general communicate with other parts of the aircraft, [1]. The automotive field, at first, inherited the anti-skid technology from the aeronautic world, but later developed refined and advanced techniques for active braking control, using for this purpose a rich set of sensors that allow one to reconstruct the whole vehicle dynamics, see e.g., [2].

Another relevant difference between the two contexts is that, in aircraft, the anti-skid is in general activated on all braking maneuvers, both in the case of landing and rejected take offs, i.e., those cases in which the aircraft, at the end of the strong

1 The authors are with Dipartimento di Elettronica Informazione e Bioingegneria at Politecnico di Milano, Piazza Leonardo da Vinci 32, 20133 Milano, Italy. E-mail addresses: \{name.surname\}@polimi.it.

2 M. Tanelli is also with Istituto di Elettronica e Ingegneria dell'Informazione e delle Telecomunicazioni - IEIIT CNR Corso Duca degli Abruzzi 24, 10129 Torino, Italy.

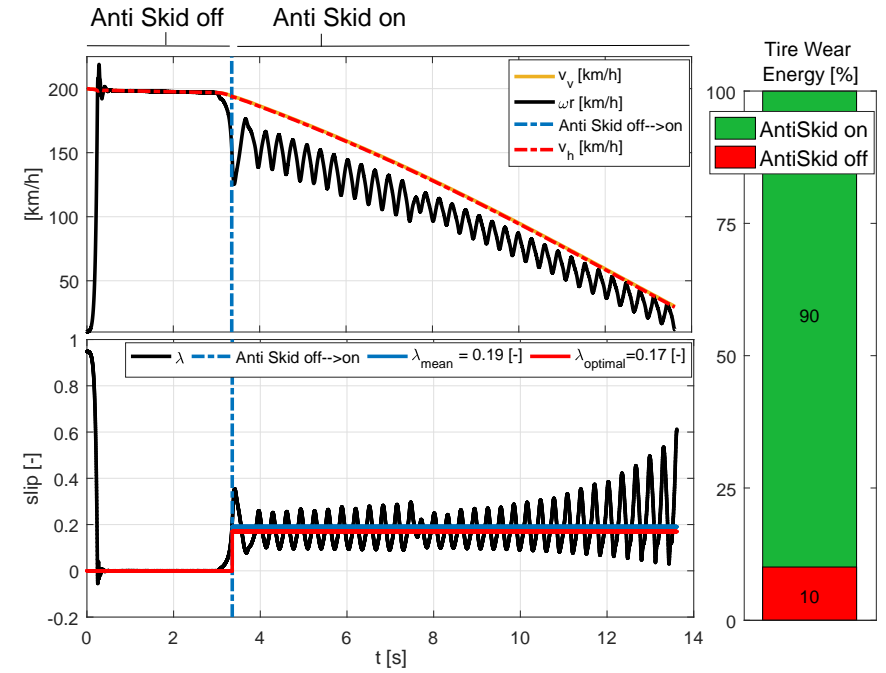

Fig. 1. Landing manoeuvre with deceleration-based anti skid controller and related tire wear contributions.

acceleration preceding the take-off, has to unexpectedly to reject it and come to a halt. Moreover, once activated, the braking controller remains active for the most part of the braking maneuver, until the aircraft reaches a nearly standstill condition. This means that the wheel skid induced by the controller has a long time over which it acts continuously on the wheels, consequently inducing a significant consumption of the tires. In turn, this makes the cost of changing tires that reach their end of life one of the most relevant in aircraft maintenance.

To fix the scope of the considered problem, let us refer to Figure 1, which shows on the top the different phases of an aircraft landing, and, on the bottom, a possible corresponding time history of speed and skid, assuming that an anti-skid controller is in place. Of course, being interested in investigating the impact of the anti-skid control on tire wear, one wants first to ascertain that the amount of energy that is actually involved in the controlled braking phase is indeed much larger than that spent in the touch-down time interval. The right part of Figure 1 shows that the energy share of the two phases is of approximately $10 \%$ for the touch down (when the still wheels are abruptly put in contact with the ground and experience a very strong skid once they feel the very large forward speed induced by the aircraft inertia at the beginning of the wheelon-ground phase) and $90 \%$ for the braking phase itself. This allows us to claim that, in case we can relate the anti-skid tuning with the resulting tire-wear, then we have a means of significantly varying the tire consumption by acting on the controller itself. Of course, to be of interest, such a reduction 


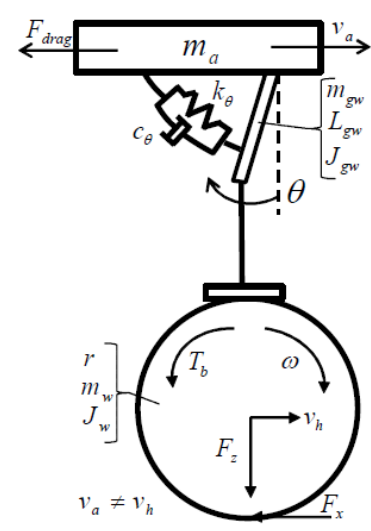

Fig. 2. Schematic view of an aircraft landing gear dynamic model.

in consumption must not result in an overly degradation of the braking performance.

This work focuses on such an analysis, providing a dynamic model of the tire consumption mechanism that can be used during braking, and then studies the interplay between antiskid control and tire wear. Specifically, two different control algorithms are considered. One is in line with the current industrial practice, being designed using only the measurement provided by the wheel speed sensor, and yielding a final cyclic skid behavior such as that shown in the bottom part of Figure 1, see e.g., [3]. The second one is a more advanced anti-skid control approach, which uses a measure or an estimation of the aircraft velocity and designs a genuine skid regulation system, see e.g., [4]. Our analysis will show that the main driver in moving from the traditional to the new design approach is to be found in the much increased capability in acting on the tire wear phenomenon. The obtained results lead to the idea that tire consumption can actually be controlled via anti-skid control design, which is demonstrated in this paper. This concept, and its further elaboration, have been recently protected with a patent application, [5].

In this paper, we offer, to the best of our knowledge, the first detailed contribution to tire wear modelling for aircraft, introducing a dynamic description of this phenomenon that allows us to capture the importance of its close relationship with anti-skid braking. This work leverages a first brief description of these results currently submitted to the 2019 ECC, [6]. The conference version of the paper presents a limited part of the material contained in this manuscript. In particular, the major extension of the journal version is the presentation of the experimental validation, paired with more detailed modelling results.

The rest of the paper is structured as follows: Section II presents the landing gear dynamics, while Section III outlines the dynamic tire consumption model proposed in this work. Further, Section IV briefly introduces the considered anti-skid control algorithms and Section V performs the combined analysis of anti-skid control and tire consumption. Finally, Section VI shows how one can directly control tire-consumption and obtain a safe braking at the same time.

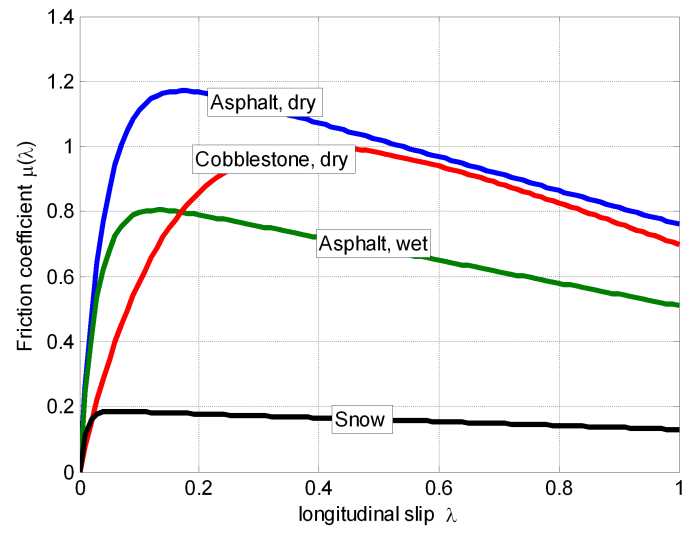

Fig. 3. Burckhardt model of the friction coefficient $\mu(\lambda)$

\section{BRAKING DYNAMICS MODEL}

As a basis for anti-skid design in aircraft, let us consider the dynamics of the landing gear, assuming that the mass insisting on it is that of approximately half aircraft. Such condensed modelling is motivated by the fact that industrial anti-skid control systems are embedded in the single braking system, each installed on a single landing gear, see e.g., [7], [8]. Such model is the aeronautic counterpart of the so-called single-corner model used for braking control of vehicles, [2]. To obtain a complete model of the landing gear for anti-skid control problems, the wheel dynamics must be described; to this end, the wheel slip ratio is defined as

$$
\lambda=\frac{v_{h}-\omega r}{v_{h}}
$$

where $v_{h}$ is the wheel hub speed, $r$ is the wheel radius and $\omega$ is the angular wheel speed. The hub speed is defined as

$$
v_{h}=v_{a}-\dot{\theta} L_{g w},
$$

where $v_{a}$ is the aircraft longitudinal speed, $\dot{\theta}$ is gear walk angular speed and $L_{g w}$ is the length of the link between the chassis and the wheel. The tire-runaway interaction is defined by the longitudinal force $F_{x}$ expressed as a function of both vertical load and wheel slip in the form

$$
F_{x}=F_{z} \mu(\lambda) \text {. }
$$

In this formulation, $\mu(\lambda)$ is the longitudinal friction coefficient, which is a function of the wheel slip and describes the available friction depending on the runaway surface. Various empirical analytical expressions of $\mu(\lambda)$ were proposed in the literature. A widely-used expression (see e.g., [2], [9]) which we borrow from the automotive context is the so-called Burckhardt model

$$
\mu(\lambda)=\vartheta_{1}\left(1-e^{-\lambda \vartheta_{2}}\right)-\lambda \vartheta_{3}
$$

where the description of the road surface is given by $\vartheta_{i}$, $i=1,2,3$. Different values of these parameters allow to model different tire-road friction conditions; the curve $\mu(\lambda)$ in different conditions is displayed in Figure 3.

The main difference between the control-oriented description of the landing gear and the automotive single-corner 
model is the presence of the so-called gear walk phenomenon. Such a phenomenon can be described as an oscillatory motion of the landing gear in the longitudinal direction taking place about a static vertical center line. This motion is due to the interaction between tire and runaway modulated by the vertical load which deflects the landing gear. Such an oscillation may of course interact with the anti-skid closed-loop behavior, and must be considered in the design of anti-skid systems for aircraft, see e.g., [1], [8]. The control-oriented view of the gear-walk phenomenon in a landing gear can be compactly described as a rotational spring-damper, as shown in Figure 2.

In the modeling of the landing gear, a constant wheel radius and static vertical load are considered. Both assumptions come from the single-corner approach, the starting point for this paper, which does not take into account the suspension dynamics. In the automotive field, neglecting the suspension dynamics during the braking control system design, results in only a retuning of the controller parameters; this takes into account the load transfer dynamics and it generally produces different controller parameters for the front and rear wheels. This approach relies upon the fact that wheel dynamics are sufficiently faster than chassis and suspension excitation frequencies. To our best knowledge, the combination of the gear-walk, suspensions and longitudinal motion has not been extensively studied in the aeronautic field from the anti-skid design point of view yet. In principle, the gear-walk motion can introduce a strong coupling between the longitudinal and vertical dynamics.

The non-linear model of the aircraft landing gear, which is schematically represented in Figure 2, can be compactly described as

$$
\begin{aligned}
& \left(m_{a}+m_{w}+m_{g w}\right) \dot{v}_{a}-J_{\theta} \ddot{\theta}=-F_{x}-F_{d r a g} \\
& J_{\ddot{\theta}} \ddot{\theta}-J_{\theta} \dot{v}_{a}+c_{\theta} \dot{\theta}+k_{\theta} \theta=L_{g w} F_{x} \\
& J_{w} \dot{\omega}=r F_{x}-T_{b},
\end{aligned}
$$

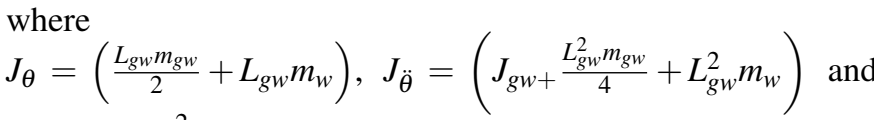
$F_{\text {drag }}=\alpha_{d} v_{a}^{2}$ is the drag force. The parameter $\alpha_{d}$ is tuned based on experimental data that are not reported here for sake of brevity.

Finally, based on experimental data, see [10], the actuator dynamics were modeled with the second order transfer function

$$
A(s)=\frac{\omega_{n}^{2}}{s^{2}+2 \xi_{n} \omega_{n} s+\omega_{n}^{2}},
$$

with $\xi_{n} \approx 0.7$ and $f_{n}=\frac{\omega_{n}}{2 \pi} \approx 15 \mathrm{~Hz}$, which is used in the simulation setting.

\section{TIRE WEAR MODELLING}

The tire wear phenomenon is due to several mechanisms ranging from mechanical delamination to rubber oxidation [11], [12]. It is strongly non-linear, and it is influenced by numerous variables, such as contact geometry, the presence of contaminants between contact surfaces, tire temperature and pressure, to name the most important. Furthermore, see also [13], [14], the most relevant dynamic factors are the

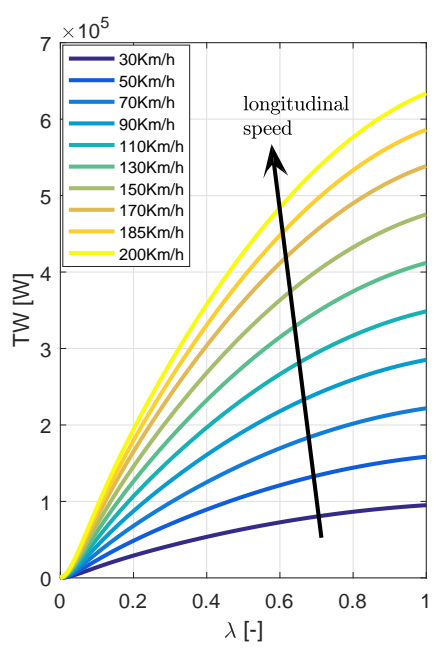

(a)

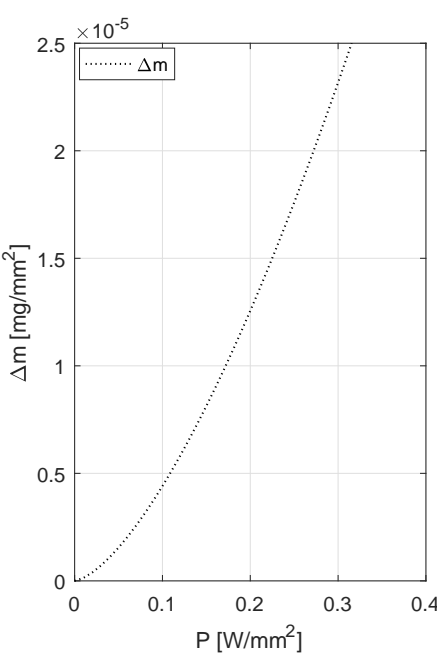

(b)
Fig. 4. Tire-wear power as a function of the wheel slip for different values of the longitudinal speed (a); Tire mass-loss as a function of the tire-wear power (b).

longitudinal vehicle speed, the longitudinal slip and the tire side-slip angle, i.e., the angle between the tire longitudinal axis and the direction of the tire longitudinal speed. As in this work the focus is on longitudinal braking maneuvers, such as those performed during landings and rejected take-offs (RTOs), in what follows the tire side-slip angle can be considered null, as tire longitudinal axis and tire speed are aligned.

A suitable analytical tire wear model can be now derived: from a physical viewpoint, tire wear is generated by the difference between the longitudinal frictional power $F_{x} v$ and the braking power $T_{b} \omega$; this second term can be obtained from the rotational wheel dynamics in (7). Tire wear power $(T W)$ is then defined as follows:

$$
T W=F_{x}\left(v_{h}-\omega r\right)+J_{\omega} \omega|\dot{\omega}|=F_{x} v_{h} \lambda+J_{\omega} \omega|\dot{\omega}| .
$$

Note that the second term represents the wear contributions due to the typical wheel speed oscillations induced by the ABS control algorithm, see [3], [15], and it is typically disregarded in the automotive literature, [14]. This is consistent with the fact that, in the automotive context, the ABS is very rarely activated and the main reason behind tire wear is the consumption over a considerable amount of travelled distance, with negligible contributions coming from the wheel deceleration. On the other hand, in the aeronautical world, tire wear is mostly related to the braking manoeuvres in a landing/RTO during which ABS is always activated: by means of the second term in (9), significant wheel speed oscillations introduced by the anti-skid itself can be included in the analysis of the tire wear phenomenon.

In Figure 4a, a steady-state sensitivity analysis of (9) is shown. By inspecting the left figure, one has confirmation of the physical interpretation of the tire wear phenomenon described above: as wheel slip and longitudinal speed increase, a larger tire wear power is generated, thus increasing the overall tire consumption. 


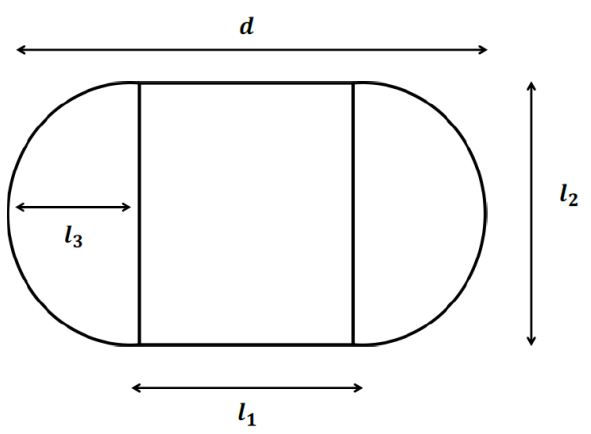

Fig. 5. Tire Wear: tire contact area.

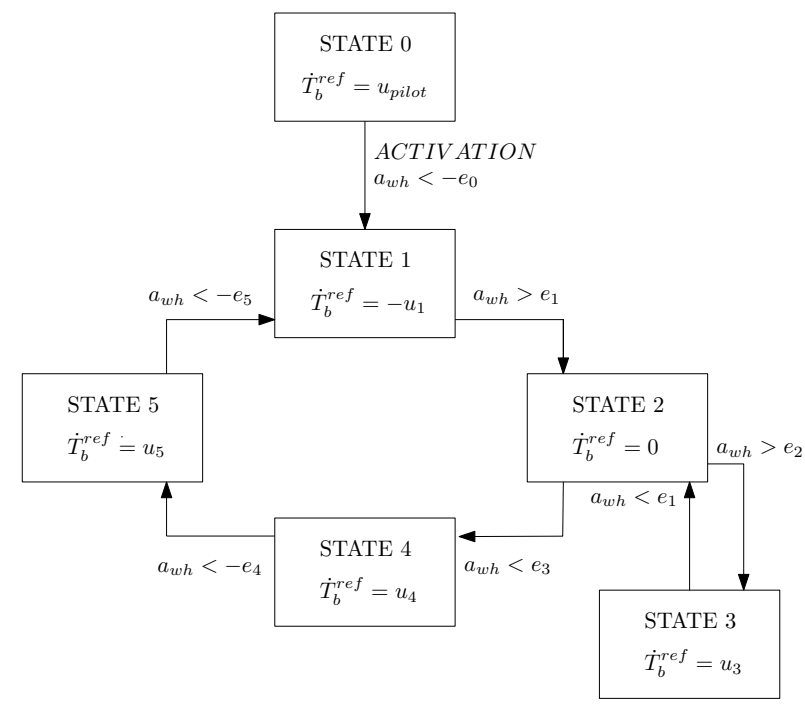

Fig. 6. Finite state machine representing the basic version of the 5-phase braking control algorithm in [15].

Furthermore, according to [11], [13], the mass loss per unit contact area can be computed as

$$
\begin{gathered}
\Delta m=f_{1} P^{f_{2}} \quad\left[\mathrm{mg} / \mathrm{mm}^{2}\right] \\
\Delta M=\Delta m l_{2} \Delta S, \quad[\mathrm{mg}]
\end{gathered}
$$

where $P=T W / a_{\text {cont }}$ and $a_{\text {cont }}$ is the contact area between tire and road, see Figure 5. From (10), the total mass loss during the braking manoeuvre can be computed as in (11) where $l_{2}$ is the tire width and $\Delta S$ is the stopping distance. In Figure $4 \mathrm{~b}$, the results obtained using Equation (10) are reported.

\section{STRUCTURE OF THE ANTI-SKID CONTROLLERS}

In order to study the interplay between tire wear and anti-skid control, we now introduce the two braking control approaches that will be used in the following analysis. Specifically, the model of a deceleration-based controller that induces a limit-cycle behaviour on the wheel skid is first introduced, followed by another approach that directly controls the wheel skid.

\section{A. Traditional anti-skid control approach}

In order to formally describe the working principles of a commercial aeronautical anti-skid that are based upon the wheel speed measurement only, a deceleration-based control algorithm must be employed. For the design of such an algorithm we leveraged on the work first presented in [15], where a threshold-based control algorithm was proposed, based on the wheel acceleration, that allows achieving a stable limit cycle on the wheel slip, which in principle should position itself around the optimal value of the wheel slip for the given friction condition, which of course is assumed to be unknown. Such an algorithm is referred to as 5phase-ABS, and its finite state machine representation is shown in Figure 6.

As can be seen, the algorithm, based on thresholds on the wheel acceleration $a_{w h}$, computed by appropriately differentiating the measured wheel speed, imposes a value to the time derivative of the braking torque $\dot{T}_{b}^{\text {ref }}$, which can be either zero, or positive or negative. According to its value, the torque in the given phase of the algorithm will be held constant, increased, or decreased, respectively. The alternation of such actions in the five different phases allows obtaining a limit cycle of the wheel slip which, with the given basic version of the algorithm, is proved to enjoy stability properties. When such an algorithm is to be implemented in a real system, where actuator dynamics and measurement noises must be taken into account, the basic version must be modified as depicted in Figure 7, in order to preserve the desired features.

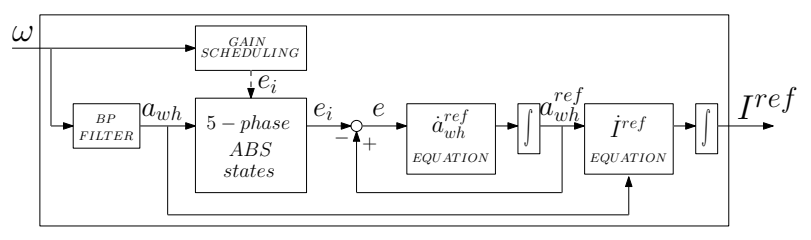

Fig. 7. Complete architecture of the 5-phase anti-skid controller

Specifically, to experimentally validate the presented control logic, we had to adopt some of the robustification approaches already present in the automotive literature and to add new ones for the specific application considered herein. We mainly refer to the work presented in [16], where several extensions of the basic algorithm are described to cope with a realistic experimental setting. In this work, we applied the closed-loop acceleration control method, the addition of which to the basic 5-phase algorithm allows us to avoid that the acceleration moves too far away from the thresholds during online functioning, as this, coupled with the actuator and other possibly unmodelled dynamics, might destroy the limit cycle and lead to an unstable closed-loop behaviour.

The overall control scheme depicted in Figure 7 is now described in detail. The wheel acceleration $a_{w h}$ is computed by numerical differentiation of the wheel speed $\omega$ represented in the frequency domain by the band-pass filter (see BP filter block). The reference braking torque is then computed based on the tracking error of the wheel acceleration, i.e.,

$$
\dot{T}_{b}^{r e f}=K\left(a_{w h}-a_{w h}^{r e f}\right)
$$


where $K$ is a suitable tuning control parameter and $a_{w h}^{r e f}$ is the set-point wheel acceleration obtained by integrating the following reference signal

$$
\dot{a}_{w h}^{r e f}= \begin{cases}-\operatorname{sign}(e) \sqrt{2 u_{p}|e|} & \text { if } \sqrt{2 u_{p}|e|}<v_{p} \\ -\operatorname{sign}(e) v_{p} & \text { if } \sqrt{2 u_{p}|e|} \geq v_{p},\end{cases}
$$

where $v_{p}$ and $u_{p}$ are constant tuning parameters and $e=a_{w h}^{r e f}-$ $e_{i}$, with $e_{i}$ being the threshold on the acceleration set in phase $i$ of the algorithm, see again Figure 6. The main idea is to define a suitable rate of change of $a_{w h}^{r e f}$ to take into account the physical limitations and the delay introduced by the actuator dynamics.

Besides such internal loop, we introduced a novel scheduled variation of the thresholds values $e_{i}$. This is needed to manage the very wide range of speed that is experienced in aircraft braking, which starts from approximately $200 \mathrm{~km} / \mathrm{h}$ and proceeds with a single, long braking manoeuvre, which does not end till the vehicle is at standstill or at very low speed. Specifically, the adaptation is obtained letting

$$
\begin{aligned}
& g_{e_{i}}= \begin{cases}g_{e_{i}, 0} & \text { if } \omega>\omega_{0} \\
g_{e_{i}, j} \quad \text { if } \omega_{n}<\omega \leq \omega_{0} \quad j \in[1, n] & \text { if } \omega \leq \omega_{n} \\
g_{e_{i}, n} \quad \text { if }\end{cases} \\
& g_{e_{i}, j}=\frac{g_{e_{i}, j}-g_{e_{i}, j-1}}{\omega_{j}-\omega_{j-1}}\left(\omega-\omega_{j-1}\right)+g_{e_{i}, j-1} \\
& e_{i}=g_{e_{i}} \bar{e}_{i},
\end{aligned}
$$

\begin{abstract}
where a proportional scheduling of the threshold values $e_{i}$ as a function of the measured wheel speed $\omega$ is expressed. The main idea is to smoothly reduce the nominal values of the acceleration thresholds $\bar{e}_{i}$ proportionally to the variation of the aircraft speed in order to decrease the amplitude of the acceleration oscillations as speed decreases. Specifically, the whole wheel speed range was subdivided into $n$ sectors whose extremal values of the $j$-th sector are defined by $\left[\omega_{j-1}, \omega_{j}\right]$. It is worth recalling that, see for example [17], all active braking systems become unreliable at low speed, both for the wheel slip dynamics getting infinitely fast as the speed goes to zero, and to the increasing degradation of the wheel speed measurement. To deal with these issues, alternative open-loop schemes are used for taking vehicles from low speed values to standstill.

A typical closed-loop behaviour of such controller is depicted in the left plot of Figure 1, which shows that the wheel slip indeed evolves on a limit-cycle, and that such a cycle is almost centered around the (unknown) optimal value of the slip for the given road condition, dry road in the case show in the figure. By computing the average wheel slip achieved in the braking maneuver, indicated with $\lambda_{\text {mean }}$, in the case shown in Figure 1 we obtained $\lambda_{\text {mean }}=0.19$. Note that $\lambda_{\text {mean }}$ will be the variable of interest to define the tire-wear associated to the braking maneuver.
\end{abstract}

\section{B. Slip control}

As for the design of the proposed slip controller, the adopted control scheme is reported in Figure 8. To better understand

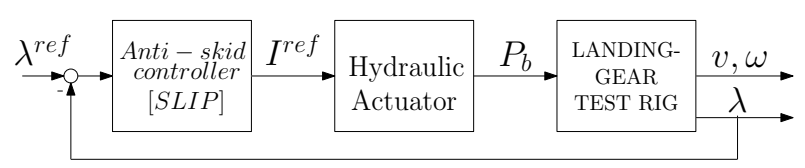

Fig. 8. Structure of the wheel slip controller

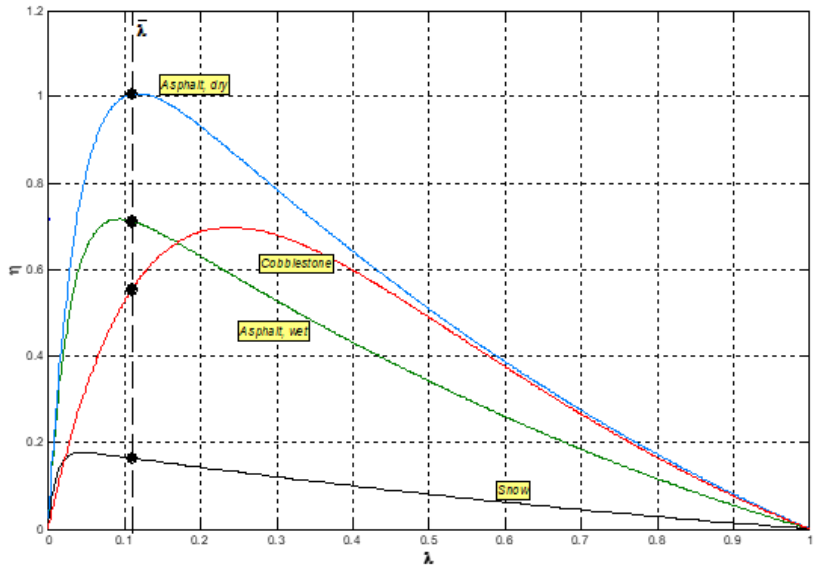

Fig. 9. Pictorial representation of the wheel slip control algorithm in the $\lambda, \eta$ domain.

the design rationale behind the wheel slip controller, let us first define the normalized wheel deceleration as

$$
\eta=-\frac{\dot{\omega} r}{g}
$$

where $\dot{\omega}$ is the wheel deceleration and $g$ is the gravitational acceleration. Such a variable allows one to compare directly the wheel deceleration to that of the body of the aircraft expressed in $\mathrm{g}$. In a linearized context, it is possible to pictorially describe the slip control principle in the $(\lambda, \eta)$ domain using the representation shown in Figure 9. This figure shows, for each considered road condition, the $(\eta, \lambda)$ equilibrium manifolds obtained from the linearized equations of the single corner model. The vertical solid line defines the set-point value of the wheel slip to be tracked by the slip control system. As can be seen, a single closed-loop equilibrium point exists for each road surface and for each choice of the set-point value, and a good performance trade-off can be obtained with a fixed choice of such a set-point value on all friction conditions.

The tuning of the controller parameters was been carried out based on the linearization of the presented non-linear model around a suitable equilibrium point (see Appendix VIII for the complete formulation of the linearized system): a low speed value has been chosen to stabilise the system where it is more challenging due to its faster dynamics. Then, classical loopshaping techniques were applied, designing an LTI controller with phase margin $\varphi_{m} \approx 50^{\circ}$ and a closed-loop bandwidth of approximately $12 \mathrm{~Hz}$. As previously discussed, in such formulation, the gear-walk dynamics is considered in the landing gear modelling and, thus, it is also taken into account during controller tuning. The gear-walk dynamics introduces a resonance in the frequency response at approximately $8 \mathrm{~Hz}$, within the typical anti-skid controller bandwidth and, thus, it could negatively affect the closed-loop performance. 

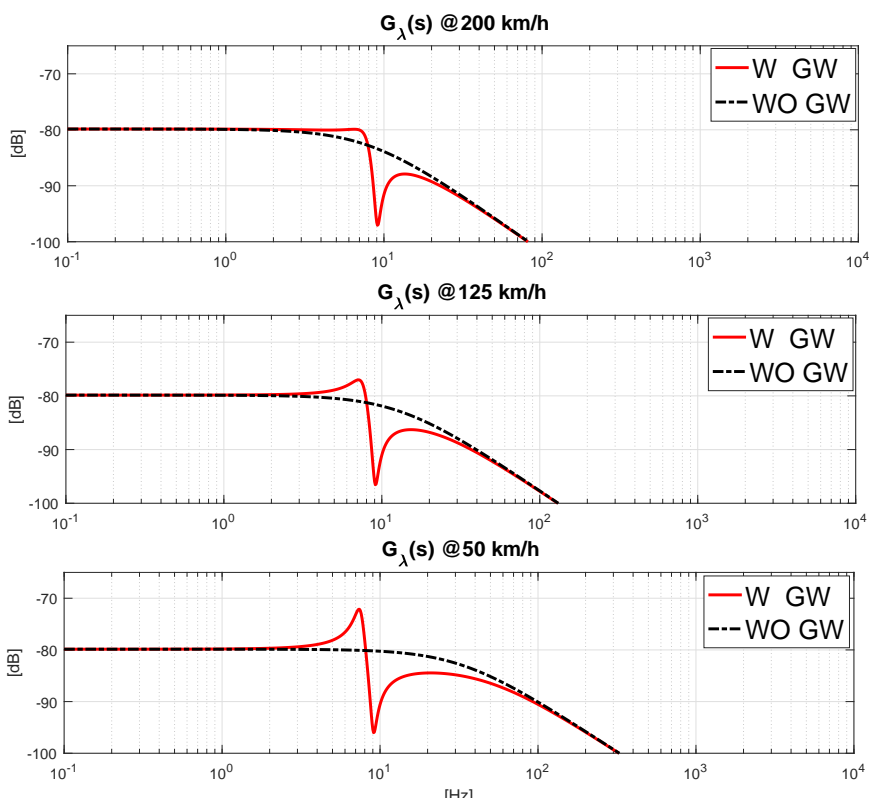

Fig. 10. Sensitivity of the frequency responses $G_{\lambda}(j w)$ to different aircraft speed values, with (solid red line) and without (dashed-dot black line) the gear-walk dynamics.

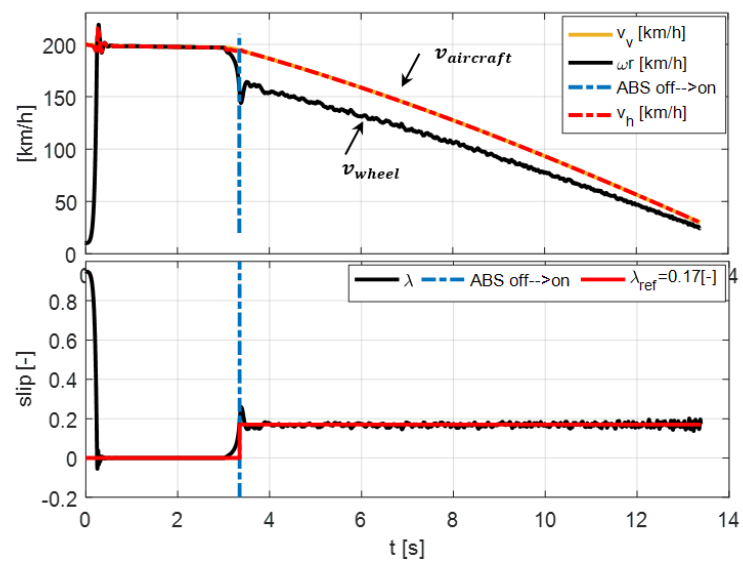

Fig. 11. Closed-loop behaviour of the slip control system on dry road. Top plot: time histories of aircraft and wheel speed; bottom plot: time histories of the wheel slip.

To appreciate the effects of the gear-walk phenomenon, the frequency responses associated with the transfer function from braking torque to wheel slip $G_{\lambda}(s)$ were computed for different speed values. Specifically, Figure 10 shows the comparison of the two frequency responses with and without the gear-walk dynamics, considering different and constant values of aircraft speed.

A typical closed-loop behaviour of the slip controller on dry road is shown in Figure 11 proving that the wheel slip indeed evolves toward the desired set-point value, $\lambda_{\text {ref }}=0.17$ in the case depicted in the figure.

The following section will relate the tire consumption with the wheel slip set-point value, assuming that the latter will be reached and maintained during the maneuver thanks to the action of the slip controller.

\section{Remark}

In the ground-vehicles braking control literature, continuous control algorithms which are based also on deceleration measurements have been proposed, see, for example the so-called Mixed Slip-Deceleration Control, [2], [18], which mixes, in an LTI setting, wheel slip and linear wheel deceleration to build a new control variable, or the approach presented in [19]. For sure, continuous and hybrid/switching braking controllers have different pros and cons, as highlighted for example in [20], which considered different control algorithms and analyzed their performance using the stopping distance as main metric. In this work, we selected the two aforementioned controllers taking one from each of the two families, in the spirit of comparing a control approach that is mainstream in anti-skid for aircraft (the 5-phase one) to a slip-based one, currently not in use in the field, to demonstrate that moving towards a slip-based approach could open new possibilities in tire consumption control. Once this novel approach is accepted, and we hope this work will help to achieve this, then indeed many other control approaches can be tested to see which handles the trade-off optimally under different perspectives.

\section{TIRE-WEAR ANALYSIS: SENSITIVITY TO ANTI-SKID BRAKING}

Based upon the modelling of the tire wear phenomenon described in Section III, it is now possible to evaluate and compare the tire mass-loss obtained with both the anti-skid control logics described in Section IV. To concisely describe the performance of the closed-loop system, the stopping distance $\Delta S$ is considered as a relevant and easily definable cost function.

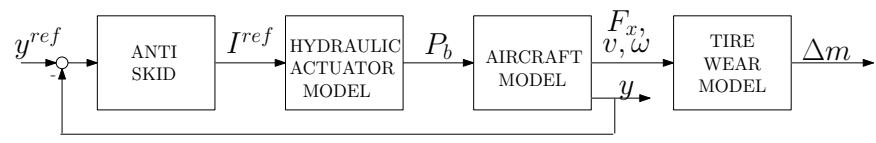

Fig. 12. Tire Wear: schematic view of the sensitivity analysis to anti-skid control with computation of the tire consumption in terms of mass-loss $\Delta m$.

For this analysis, the scheme depicted in Figure 12 is adopted, where the tire wear model has been obtained based upon the results of Section III. The aircraft and actuator dynamics are those described in Section II; a generic antiskid block is reported in order to be representative of both control strategies analysed in Section IV.

To explore the trade-off between performance, expressed in terms of stopping distance $\Delta S$, and tire mass-loss $\Delta m$, different values of the wheel slip set-point have been considered for the slip control algorithm. Specifically, braking maneuvers have been carried out for values of $\lambda^{r e f}$ ranging from 0.01 to 0.17 : the lowest to analyse the effect of almost no slip from the tire wear point of view; the largest corresponding to the peak of the friction curve implemented in the aircraft simulator, and used to analyse the effect on tire wear of a control logic aiming to exploit all the available tire-road grip.

The overall results are reported in Figure 13, where Figure 13a refers to the normalised mass loss $\Delta m$ of equation (10) 


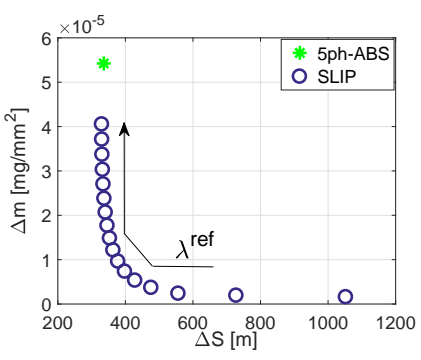

(a) Tire wear: $\Delta m(10)$

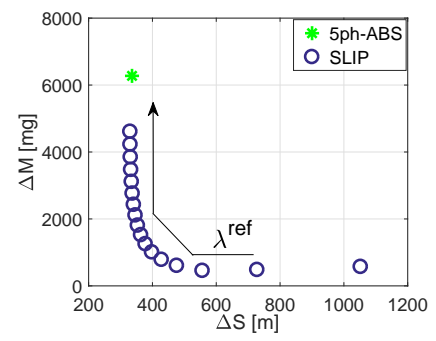

(b) Tire wear: $\Delta M(11)$
Fig. 13. Results of the sensitivity analysis of tire-wear to anti skid control

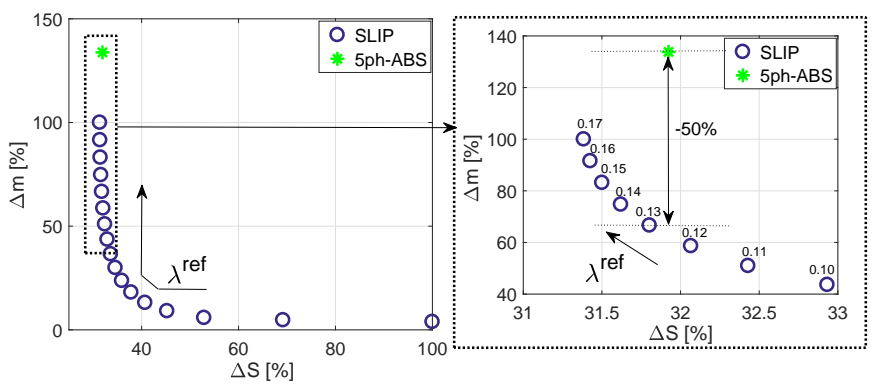

Fig. 14. Percentage variation of $\Delta m$ : zoom

while $13 \mathrm{~b}$ refers to the mass loss $\Delta M$ of equation (11). The horizontal axis represents the stopping distance $\Delta S$. Note that the difference in these two figures is only in a scale factor given by the overall covered area during the braking manoeuvre $\left(l_{2} \Delta S\right)$.

From these figures, three main results can be withdrawn: first of all, the most severe tire wear has been obtained using the 5phase-ABS. Second, a strong correlation between tire wear and the reference value of the wheel slip $\lambda^{\text {ref }}$ is observed using the pure slip controller. Consistently with the physical interpretation of this phenomenon, the nearer the reference slip value $\lambda^{r e f}$ to the one corresponding to the peak of the friction curve, the higher the tire wear level and the lower the stopping distance. This trend is graphically indicated with arrows in the direction of increasing $\lambda^{\text {ref }}$.

Consider that such an effect cannot be obtained with the 5phase control system, as its paradigm is that of not specifying a wheel slip set-point, but to automatically bring the wheel slip of the closed-loop system to cycle around its optimal value, i.e., that corresponding to the peak of the friction curve for the current runaway condition.

Most interestingly, a strong and tangible difference between slip-based and acceleration-based control approaches is revealed: looking at the results achieved with the 5-phase algorithms, in fact, one may see that absolutely reasonable and acceptable results are obtained as far as performance are concerned, but little or no degrees of freedom are available to act on tire consumption.

In fact, by tuning the controller parameters, small modifications of the resulting limit cycle may be obtained, but these in turn reflect in only very small variations of the average value of the wheel slip obtained in closed-loop, and thus minor modifications of the final tire mass-loss. Note also that such modifications can be carried out having in mind not to alter the closed-loop stability and robustness against, for example, the unknown road conditions.

Looking at the slip control approach, instead, it is apparent that a great flexibility in accommodating constraints that can optimally mix performance and tire consumption is indeed available. To better quantify this observation, the content of Figure $13 \mathrm{~b}$ was normalised and shown in Figure $14^{1}$. The main advantage of a slip control is apparent: tire wear is reduced of almost $50 \%$ with respect to the classical decelerationbased (5phase-ABS) without compromising the braking performance in terms of stopping distance. Furthermore, thanks to the significant slope of the Pareto curve, slightly reducing $\lambda^{\text {ref }}$ would allow to achieve a further tire wear reduction with an almost negligible increase in the stopping distance.

Overall, these results show that a very promising way to be able of directly and knowingly influencing the tire consumption in aircraft is to design the anti-skid control system solving a slip regulation problem. Of course, this implies that a new measurement must be made available to obtain an estimate of the aircraft speed. If connecting the main flight controller with the braking system to directly send to it the aircraft speed measurement given by the main inertial measurement unit (IMU) may pose excessive safety constraints, a landinggear-based solution can be devised placing a local IMU on the rigid part of the landing gear to get information for estimating the aircraft speed, thus avoiding critical coupling among the different subsystems.

\section{TIRE WEAR CONTROL}

We now focus on the direct control of the tire wear during the braking maneuver. In particular, starting from the relationship between the wheel slip reference $\lambda^{\text {ref }}$ and the Pareto curve in the plane $\Delta S-\Delta m$ analysed in Section $\mathrm{V}$, we leverage this information to select the most suitable reference value $\lambda^{\text {ref }}$ that ensures the desired consumption level.

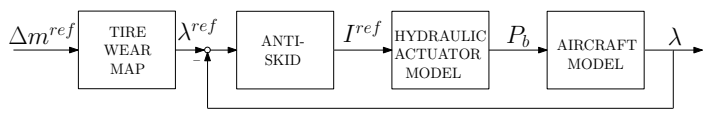

Fig. 15. Schematic view of the tire-wear control approach.

To this end, assuming for example that no too stringent constraints on the stopping distance $\Delta S$ are in play (which may come, for example, in cases of particularly short runaways), one could decide to reduce the tire consumption while maintaining sufficient braking performance. The control scheme in Figure 15 shows how to automatically define the reference value $\lambda^{\text {ref }}$ which results from the selection of a desired tire wear level expressed as a mass-loss $\Delta m^{\text {ref }}$.

For this purpose, using the Burckhardt friction curves of Figure 3, the tire wear map of both dry and wet asphalt is obtained (the dry asphalt curve is the same as in Figure 13b).

\footnotetext{
${ }^{1}$ The normalization values have been chosen among the slip control results: the horizontal axis has been normalized with respect to the highest stopping distance value (corresponding to $\lambda^{\text {ref }}=0.01$ ); the vertical axis has been normalized to the highest tire wear value $\left(\lambda^{\text {ref }}=0.17\right)$.
} 


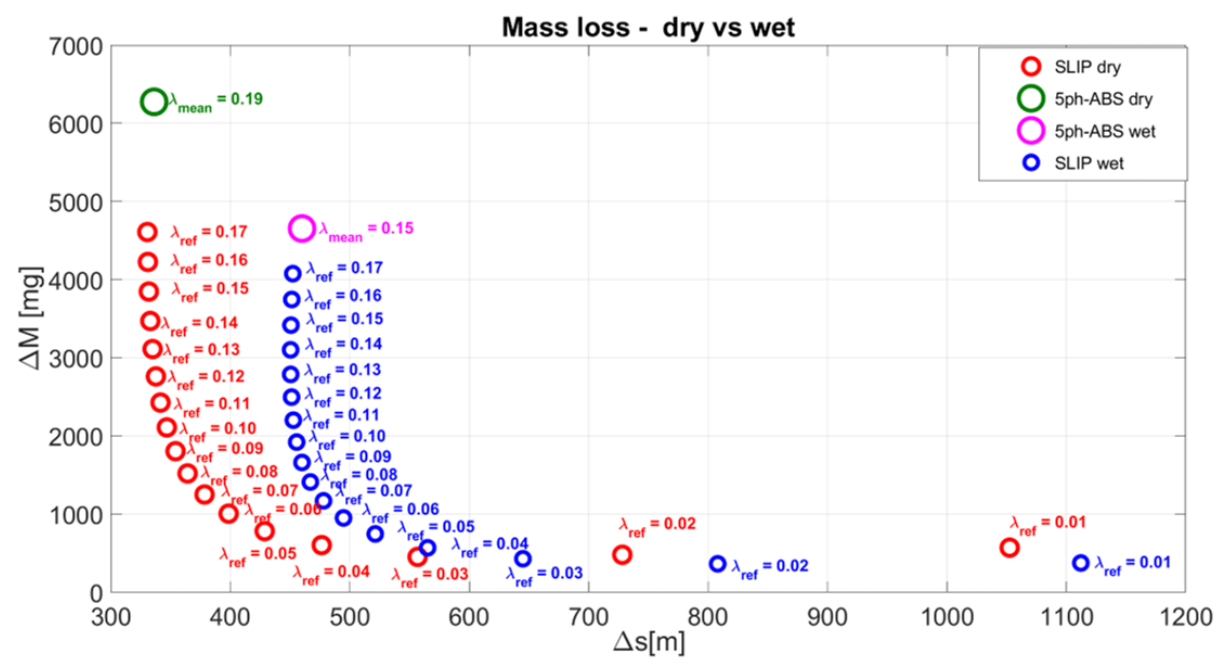

Fig. 16. Mass loss as a function of the stopping distance for varying slip reference value $\lambda^{\text {ref }}$ and varying road condition (dry and wet).

Notice that the same trend of the dry asphalt Pareto curve is maintained also in the wet asphalt road condition. Note also that, once the desired mass loss is fixed, the corresponding reference value $\lambda^{\text {ref }}$ is very similar between dry and wet asphalt road condition as reported in Table I.

\begin{tabular}{clll}
\hline$\Delta m[\mathrm{mg}]$ & $\lambda_{\text {ref }}^{\text {dry }}$ & $\lambda_{\text {ref }}^{\text {wet }}$ & $\Delta \lambda_{\text {ref }}$ \\
\hline 500 & 0.03 & 0.04 & -0.01 \\
1000 & 0.06 & 0.06 & 0 \\
1500 & 0.08 & 0.08 & 0 \\
2000 & 0.1 & 0.1 & 0 \\
2500 & 0.1 & 0.12 & -0.01 \\
3000 & 0.13 & 0.14 & -0.01 \\
3500 & 0.14 & 0.15 & -0.01 \\
4000 & 0.15 & 0.17 & -0.02 \\
4500 & 0.17 & - & - \\
\hline \multicolumn{4}{l}{ TABLE 1 }
\end{tabular}

SENSITIVITY ANALYSIS AT FIXED $\Delta M$

In order to better visualize the effect of a $\mu$-jump on the performance of tire wear control, Figures 17 and 18 show the consequence of such an event during the braking manoeuvre. In particular, before the beginning of the braking manoeuvre, $\Delta M^{r e f}=3000 \mathrm{mg}$ is selected on dry asphalt and the antiskid corresponding reference value is selected as $\lambda^{r e f}=0.127$ ). When the aircraft speed reaches $100 \mathrm{~km} / \mathrm{h}$ the $\mu$-jump event occurs and a different behaviour can be observed: Figure 17 shows the adaptation of the slip reference value to the corresponding one for wet asphalt, i.e., $\lambda^{r e f}=0.137$, while 18 shows that the initial reference value is maintained after the change of road condition. As already noted from the numerical values, a very small difference of $\lambda^{\text {ref }}$ is observed between dry and wet asphalt which means that even if the $\mu$-jump event is not detected, and thus the set-point is not adjusted, the performance are quite similar and ensure a satisfying behavior, both in terms of final mass-loss of the tire and of the braking performance.

To quantify this result and verify its generality, Figure 19 reports the overall performance of the tire wear control scheme shown in Figure 15 for different reference values $\Delta M^{\text {ref }}$. In

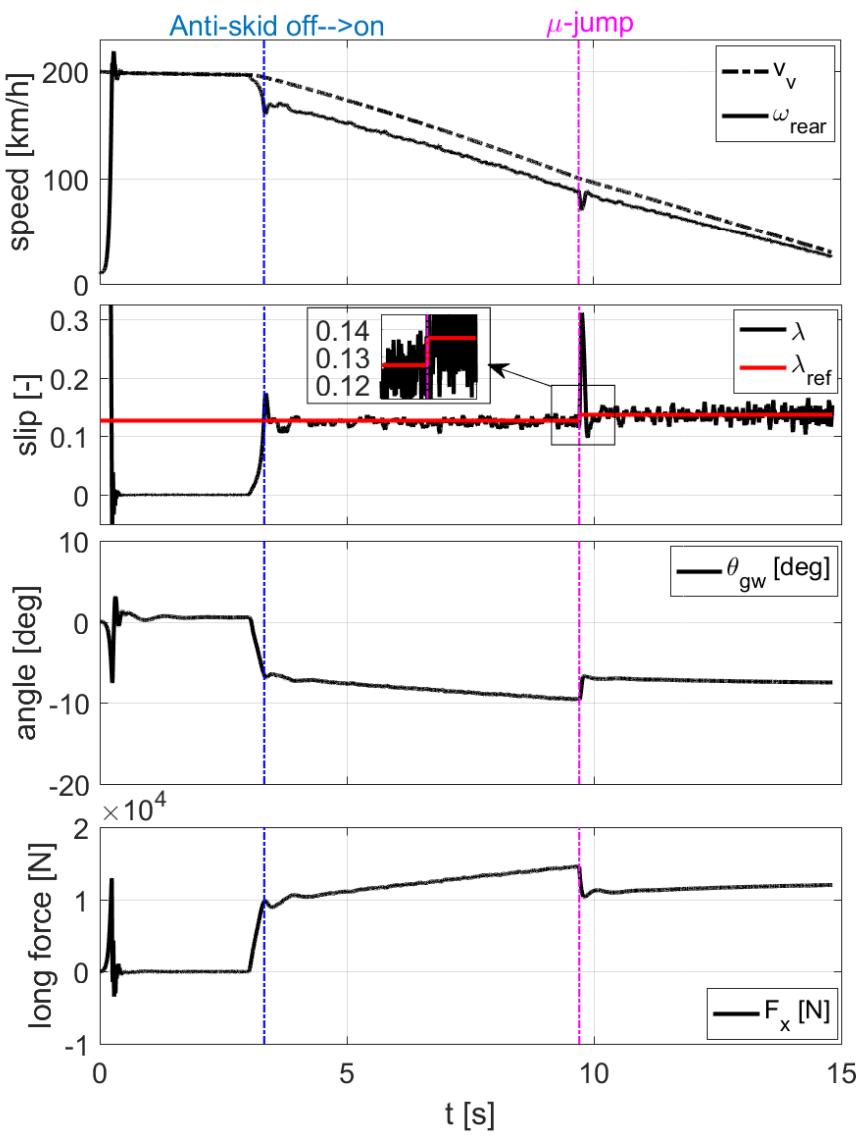

Fig. 17. Tire Wear control with $\Delta M^{r e f}=3000 m g$ with knowledge of the $\mu$ jump event Dry $\rightarrow$ Wet. From top to bottom: aircraft and wheel speed; wheel slip; gear walk position; longitudinal force.

particular, the notation $(k)$ indicates perfect knowledge of the $\mu$-jump event during the braking manoeuvre while $(n k)$ represents absence of knowledge of the the change of road condition; the ideal case is represented by the bisector of the first quadrant. As clear from this figure, a small tracking error can be related to the absence of knowledge/detection of the 


\begin{tabular}{ccccccc}
\hline & Dry & Wet & Dry to Wet (k) & Wet to Dry (k) & Dry to Wet (nk) & Wet to Dry (nk) \\
\hline$\delta e \%$ & $0 \%$ & $0 \%$ & $6.5 \%$ & $6.5 \%$ & $9 \%$ & $9 \%$ \\
\hline
\end{tabular}

AVERAGE TRACKING ERROR IN TIRE-WEAR CONTROL WITH/WITHOUT KNOWLEDGE OF THE ROAD CONDITION.
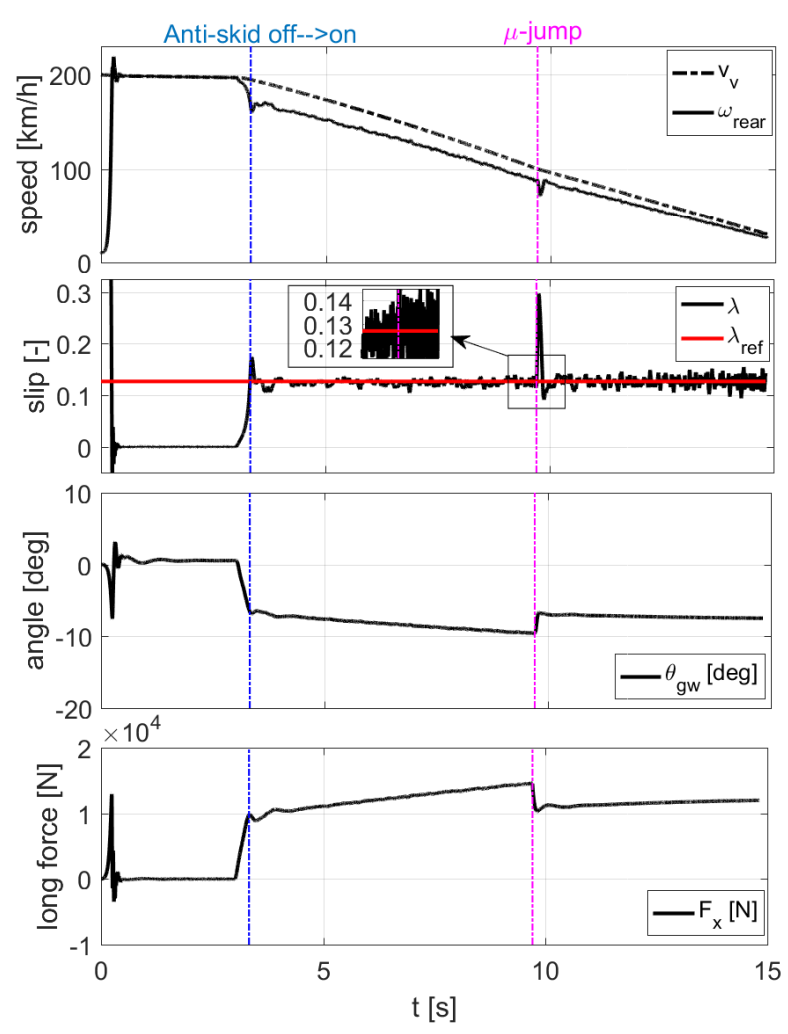

Fig. 18. Tire Wear control with $\Delta M^{r e f}=3000 m g$ without knowledge of the $\mu$-jump event Dry $\rightarrow$ Wet. From top to bottom: aircraft and wheel speed; wheel slip; gear walk position; longitudinal force.

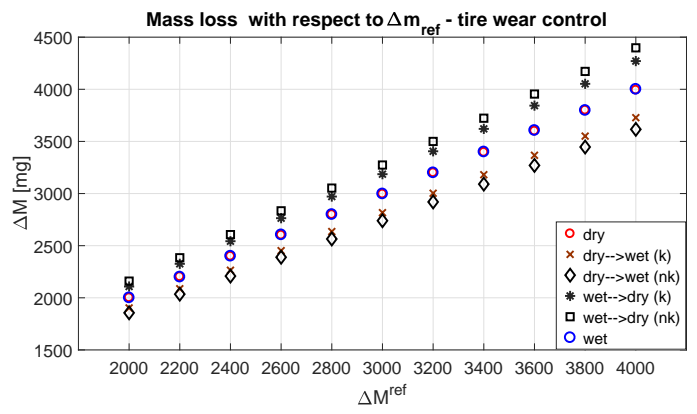

Fig. 19. Tire Wear control performance.

$\mu$-jump event.

In Table II, the percentage error

$$
\delta e=100 \times\left|\frac{\Delta m_{r e f}-\Delta m}{\Delta m_{r e f}}\right|
$$

is reported. By inspecting it, one may see that a small advantage $(6.5 \%$ against $9 \%)$ can be obtained from exact knowledge of the change in the road condition. However, even in the ideal case of immediate detection of the $\mu$-jump event it is not possible to obtain a null tracking error due to the transient time from the closed-loop equilibrium point associated to the first road condition to that linked to the second one (see again Figures 17 and 18).

\section{Remark}

It is worth noting that different road surfaces may somehow vary the numerical figures that we have investigated for the dry and wet road in this work. Anyway, we are confident that the trade-off always arises, and that a good compromise can be obtained on all types of roads. In this respect, consider also that this work targets aeronautical runaways, which can indeed have different grip levels, but in general share a rather common granularity in the underlying road surface, while the peculiarity of, for example, the cobblestone grip model with respect to the other ones in the Automotive context (see Figure (4)) lies in fact in the somehow disconnected road surface induced by the pattern of cobblestone itself. Another remark is that this optimization between tire usage and stopping distance would, in a real implementation, always made de-selectable by the pilot should any safety concern emerge, pushing back the $100 \%$ attention of the controller to achieving a minimum stopping distance, in order to cover the situations in which the road model might be out of the considered bounds for any possible reason.

\section{EXPERIMENTAL ANALYSIS}

We now proceed to perform an experimental validation of the tire wear modelling proposed in this work, together with the impact that the choice and tuning of the braking controller can have on this variable. To do this, we employ experimental data measured on a flywheel-based test rig.

\section{A. Description of the test rig}

The experimental tests presented in this work were obtained on the test rig shown in Figure 20. Specifically, the rig is composed of: a flywheel, a landing gear, a moving sledge and a loading device used to simulate the vertical load acting on the tire.

To perform the tests, the flywheel is accelerated up to the desired initial speed for the landing manoeuvre; the landing gear is detached from the flywheel in this preliminary part. Afterward, the landing gear's sledge is moved forward to the flywheel to put the wheel in contact with the flywheel and to simulate the touch-down phase that takes place before the landing manoeuvre. After a first speed transient necessary to stabilize the wheel speed in proximity of the flywheel speed, the braking manoeuvre is started by applying the desired braking pressure profile. The loading device allows to simulate the load variation due to the presence of the lift force at different aircraft speed and the consequent compression of the tire radius. 


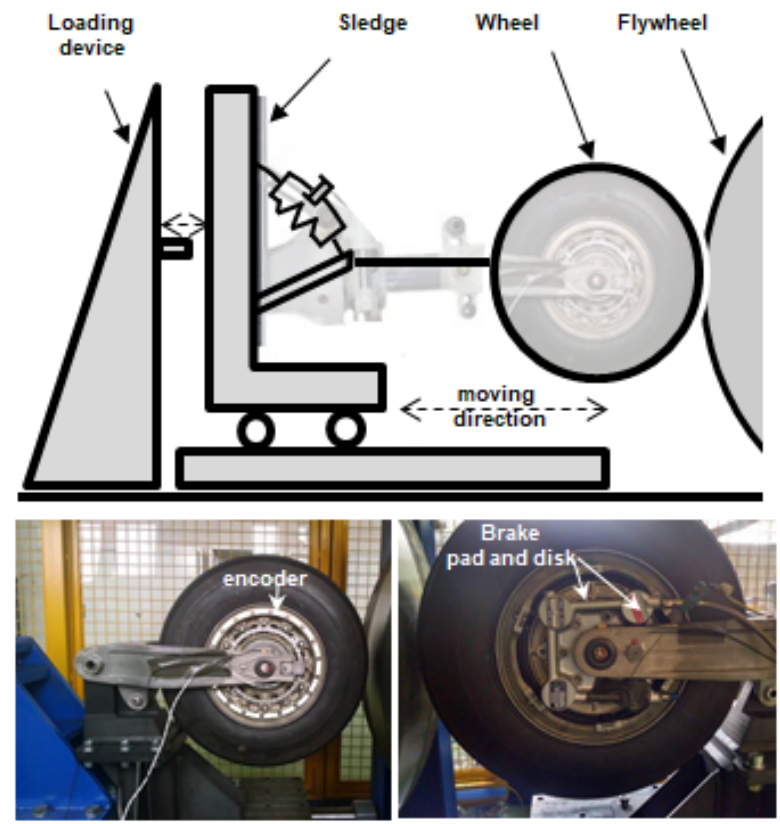

Fig. 20. Experimental set-up overview: [top] schematics of the landing-gear test rig; [bottom left] wheel encoder; [bottom right] brake pad and disk.

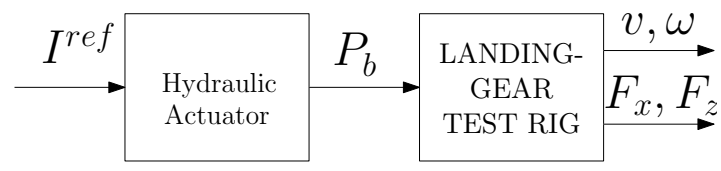

Fig. 21. Test rig: schematic overview of the experimental set-up.

The wheel and flywheel speed are measured using magnetic encoders, while a pressure sensor provides the measure of the braking pressure applied to the disk. Finally, the vertical load and the longitudinal force are measured by load cells. For the actuation of the braking pressure, an hydraulic actuator is used: a reference input current signal is imposed to obtain the desired braking pressure. For a clearer overview of the experimental set-up, in Figure 21 the test rig and the main signals are represented as a block diagram.

Based on the data measured on this rig, a tuning of the model parameters has been performed, along the lines described in [10]. After that tuning, the two controllers were tested and anti-skid braking maneuvers were carried out, thus enabling the analysis of the tire wear phenomenon in an experimental setting that is object of the next section.

\section{B. Tire wear experimental evaluation}

Based on the modelling of the tire wear phenomenon described in Section III, and using the experimental data obtained from the test rig, it is now possible to evaluate the variation of the tire mass loss due to the different behaviour of the two anti-skid controllers described in Section IV: 5phase-ABS and slip control. In particular, from the output of the closed-loop system, the mass loss $\Delta m$ was computed and expressed both as a function of the braking distance and of the tire-wear power computed from measured data.
To do this, the stopping distance was computed as

$$
\Delta S=\int_{t_{\text {Start }}}^{t_{\text {End }}} v_{\text {ground }} d t
$$

where $t_{\text {Start }}, t_{\text {End }}$ are the first and last time instant of the considered braking maneuver, respectively, and $v_{\text {ground }}$ is given by the flywheel speed measurement.

The results of this analysis are reported in Figure 22, which shows for each of the performed tests, the normalised mass loss as a function of the stopping distance (left) and of the normalised tire wear power (right), shown also in Figure $4 \mathrm{~b}$. Focusing on the tire wear performance, Figure 22 confirms that - as expected from the theoretical analysis - moving from 5phase-ABS to slip control, only small variations in the stopping distance are observed. Namely, either an improvement of $6 \%$ or a nearly an equal value $(-1 \%)$ is observed, according to the employed reference slip value. It is thus possible to confirm that almost the same braking performance can be achieved by the two proposed anti-skid approaches. However, taking also into account the effect of the anti-skid on the tire wear, the experimental results of the slip control algorithm confirm that a significant reduction in the mass loss of $-47 \%$ and $-26 \%$, respectively, can be achieved by varying the chosen set-point value. It is also worth appreciating the consistency of the slipbased control results obtained in the simulation environment reported Figure 14 and the experimental analysis shown in Figure 13. In both cases, the higher the reference wheel-slip value, the greater the mass loss due to tire wear, and the lower the stopping distance up to the point in which the Pareto curve becomes almost vertical, i.e., approximately in correspondence of the stopping distance achieved by the 5phase-ABS.
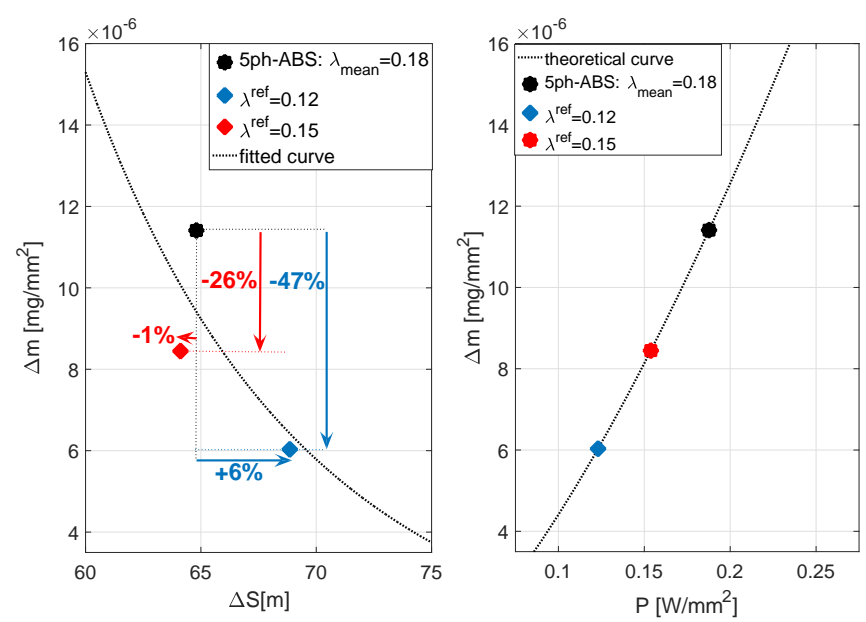

Fig. 22. Experimental validation of the tire-wear analysis: tire mass loss as a function of the braking distance (left), and tire mass loss as a function of the tire-wear power (right).

Overall, this comparison proves that a re-design of antiskid controllers for aircraft would be both cost-effective and environmentally friendly in terms of tire savings is possible and rather seamless to obtain by moving to slip-control schemes. Maintenance would be less frequent due to a lower tire deterioration exposure, and storage could be optimized due to a more deterministic and thus predictable behaviour of mass 
loss. Furthermore, slip-based approaches allow one to define the slip reference value arbitrarily, so that a lower exploitation of the available grip could be possible, i.e., imposing a reference slip value that, with only a small increase in the stopping distance, would achieve a larger reduction of the tire wear. Moreover, the tuning of the presented slip-based controllers is much easier than that of rule-based algorithms such as the 5-phase one: this could bring further cost reduction minimizing time spent for anti-skid tuning before release.

\section{CONCLUDING REMARKS}

This paper studied tire consumption in aircraft braking, presenting a model that allows computing the mass-loss experienced by a tire during anti-skid braking maneuvers. Moreover, the dynamic interplay between closed-loop braking control and tire consumption was evaluated, revealing an interested opportunity to strongly reduce tire consumption at the price of a small, and a priori predictable, increase in the braking distance, using an anti-skid control approach designed as a slip regulation problem. Based on this rationale, a tire-consumption regulation loop was designed, showing that a combined management of tire-wear levels and braking performance can be obtained rather straightforwardly. An experimental validation of the approach was also offered, confirming the proposed analysis and control approach.

\section{APPENDIX}

In this appendix, the linearized model of the landing gear dynamics given in (6) is presented, based on which the transfer functions from braking torque to wheel slip and wheel deceleration are computed. The state and input vectors are defined as follows $x=\left[\begin{array}{llll}\omega & v_{a} & \dot{\theta} & \theta\end{array}\right]^{T} ; u=\left[T_{b}\right] ; y=[\omega]$.

where: $\omega$ is the wheel speed, $v_{a}$ is the longitudinal aircraft speed, $\dot{\theta}, \theta$ are the gear-walk angular speed and position, $T_{b}$ is the braking torque.

The linearized matrices have the form

$$
\begin{gathered}
A=\left[\begin{array}{cccc}
a_{11} & a_{12} & a_{13} & 0 \\
a_{21} & a_{22} & a_{23} & a_{24} \\
a_{31} & a_{32} & a_{33} & a_{34} \\
0 & 0 & 1 & 0
\end{array}\right] \\
B=\left[\begin{array}{llll}
-1 / J_{w} & 0 & 0 & 0
\end{array}\right]^{T} \\
C=\left[\begin{array}{llll}
1 & 0 & 0 & 0
\end{array}\right]^{T}
\end{gathered}
$$

where:

$$
\begin{aligned}
& a_{11}=\bar{F}_{z} \mu_{1} \mu_{1 \omega} r / J_{w} ; \\
& a_{12}=\bar{F}_{z} \mu_{1} \mu_{1 v} r / J_{w} ; \\
& a_{13}=\bar{F}_{z} \mu_{1} \mu_{1 \dot{\theta}} r / J_{w} ; \\
& a_{21}=c_{1} \bar{F}_{z} \mu_{1} \mu_{1 \omega} ; \\
& a_{22}=c_{1} \bar{F}_{z} \mu_{1} \mu_{1 v}+2 \alpha \bar{v}_{a} d_{1} ; \\
& a_{23}=c_{1} \bar{F}_{z} \mu_{1} \mu_{1 \dot{\theta}}+c_{2} ; \\
& a_{24}=c_{3} ; \\
& a_{31}=c_{4} \bar{F}_{z} \mu_{1} \mu_{1 \dot{\theta}} ; \\
& a_{32}=c_{4} \bar{F}_{z} \mu_{1} \mu_{1 v}+2 \alpha \bar{v}_{a} d_{2} ; \\
& a_{33}=c_{4} \bar{F}_{z} \mu_{1} \mu_{1 \dot{\theta}}+c_{5} ; \\
& a_{34}=c_{6} ;
\end{aligned}
$$

$$
\begin{aligned}
& \text { den }_{c}=m_{t o t} J_{\ddot{\theta}}-J_{\theta}^{2} ; \\
& c_{1}=\left[L_{g w} J_{\theta}-J_{\ddot{\theta}}\right] / \operatorname{den}_{c} ; \\
& c_{2}=\left[-c_{\theta} J_{\theta}\right] / \text { den }_{c} ; \\
& c_{3}=\left[-k_{\theta} J_{\theta}\right] / \text { den }_{c} ; \\
& c_{4}=\left[L_{g w} m_{t o t}-J_{\theta}\right] / \text { den }_{c} ; \\
& c_{5}=\left[c_{\theta} m_{t o t}\right] / \text { den }_{c} ; \\
& c_{6}=\left[k_{\theta} m_{t o t}\right] / \text { den }_{c} ; \\
& \mu_{1}=\partial \mu /\left.\partial \lambda\right|_{\bar{\lambda}} ; \\
& \mu_{1 v}=\partial \lambda /\left.\partial v_{a}\right|_{\bar{\omega}, \bar{v}_{a}, \dot{\theta}} ; \\
& \mu_{1 \omega}=\partial \lambda /\left.\partial \omega\right|_{\bar{v}_{a}, \bar{\theta}} ; \\
& \mu_{1 \dot{\theta}}=\partial \lambda /\left.\partial \dot{\theta}\right|_{\bar{\omega}, \bar{v}_{a}, \bar{\theta}} ;
\end{aligned}
$$

\section{REFERENCES}

[1] W. E. Krabacher, "Aircraft landing gear dynamics present and future," in SAE Technical Paper. SAE International, 1993.

[2] S. M. Savaresi and M. Tanelli, Active Braking Control Systems Design for Vehicles. London, UK: Springer-Verlag, 2010.

[3] L. DAvico, M. Tanelli, S. Savaresi, M. Airoldi, and G. Rapicano, "A deceleration-based algorithm for anti-skid control of aircraft," IFACPapersOnLine, vol. 50, no. 1, pp. 14 168-14 173, 2017.

[4] L. d'Avico, M. Tanelli, S. Savaresi, M. Airoldi, and G. Rapicano, "An anti-skid braking system for aircraft via mixed-slip-deceleration control and sliding mode observer," in Proceedings of the 2017 IEEE 56th Annual Conference on Decision and Control (CDC), 2017, pp. 45034508.

[5] S. Savaresi, M. Tanelli, and L. d'Avico, "System and method for the combined control of a braking maneuver in a vehicle, in particular in an aricraft, and of the consumption of at least one tire of said vehicle." Italian Patent Application n.102018000006348, Filed on Jun 15 2018, 2018.

[6] L. D'Avico, M. Tanelli, and S. M. Savaresi, "Combining tire-wear and braking control for aeronautical applications," in Proceedings of the European Control Conference ECC, 2019.

[7] S. Gualdi, M. Morandini, and G. L. Ghiringhelli, "Anti-skid induced aircraft landing gear instability," Aerospace Science and Technology, vol. 12 , no. 8, pp. 627-637, 2008.

[8] I. Tunay, "Antiskid control for aircraft via extremum-seeking," in American Control Conference, 2001. Proceedings of the 2001, vol. 2, 2001, pp. $665-670$.

[9] U. Kiencke and L. Nielsen, Automotive Control Systems. SpringerVerlag, Berlin, 2000.

[10] L. dAvico, M. Tanelli, and S. Savaresi, "Experimental validation of landing-gear dynamics for anti-skid control design," in Proceedings of the 2018 European Control Conference (ECC-18), 2018, pp. 2751-2756.

[11] F. Braghin, F. Cheli, S. Melzi, and F. Resta, "Tyre wear model: validation and sensitivity analysis," Meccanica, vol. 41, no. 2, pp. 143-156, 2006.

[12] H. Lupker, F. Montanaro, D. Donadio, E. Gelosa, and M. Vis, "Truck tyre wear assessment and prediction," in 7th International Symposium on Heavy Vehicle Weights \& Dimensions, Delft, The Netherlands, 2002.

[13] Y. Li, S. Zuo, L. Lei, X. Yang, and X. Wu, "Analysis of impact factors of tire wear," Journal of Vibration and Control, vol. 18, no. 6, pp. 833-840, 2012.

[14] P. J. Bergmiller, Towards functional safety in drive-by-wire vehicles. Springer, 2015

[15] W. Pasillas-Lepine, "Hybrid modeling and limit cycle analysis for a class of five-phase anti-lock brake algorithms," Vehicle System Dynamics, vol. 44 , no. 2 , pp. $173-188,2006$.

[16] M. Gerard, W. Pasillas-Lépine, E. De Vries, and M. Verhaegen, "Improvements to a five-phase abs algorithm for experimental validation," Vehicle System Dynamics, vol. 50, no. 10, pp. 1585-1611, 2012.

[17] S. M. Savaresi and M. Tanelli, Active braking control systems design for vehicles. Springer Science \& Business Media, 2010.

[18] S. Savaresi, M. Tanelli, and C. Cantoni, "Mixed Slip-Deceleration Control in Automotive Braking Systems," ASME Journal of Dynamic Systems, Measurement and Control, vol. 129, no. 1, pp. 20-31, 2006.

[19] F. Cheli, A. Concas, E. Giangiulio, and E. Sabbioni, "A simplified abs numerical model: Comparison with hil and full scale experimental tests," Computers \& Structures, vol. 86, no. 13-14, pp. 1494-1502, 2008.

[20] Z. Shida, R. Sakurai, M. Watanabe, Y. Kano, and M. Abe, "A study on effects of tire characteristics on stop distance of abs braking with simplified model," in Proceedings of the International Symposium on Advanced Vehicle Control, Loughborough (UK), 2010. 